\title{
Experimentando a Influência dos Traços de Personalidade do Modelo Big Five na Recomendação de Recursos Educacionais
}

\author{
Janderson Jason B. Aguiar', Joseana M. F. R. de Araújo ${ }^{1}$, Evandro de B. Costa ${ }^{2}$ \\ ${ }^{1}$ Universidade Federal de Campina Grande (UFCG), Campina Grande - PB - Brasil \\ ${ }^{2}$ Universidade Federal de Alagoas (UFAL), Maceió - AL - Brasil \\ janderson@copin.ufcg.edu.br, joseana@computacao.ufcg.edu.br, evandro@ic.ufal.br
}

\begin{abstract}
Recent research has considered the use of the theory of Personality Traits to build the user profiles in Recommender Systems. This paper presents an experimental study of the influence of the five traits of the Big Five model in the construction of user profiles in Educational Recommender Systems. Particularly, we analyzed the possibility of using only some traits to simplify this construction while maintaining accurate personalized recommendations. Although it has not been possible to highlight precisely the traits of the Big Five model with the most significant influence on the recommendation, the results showed that the five traits do not have the same influence, and there are indications that the use of the trait Openness to Experience is sufficient.
\end{abstract}

Resumo. Pesquisas recentes têm usado a teoria dos Traços de Personalidade para construir o perfil dos usuários em Sistemas de Recomendação. Neste artigo, é apresentado um estudo experimental sobre a influência dos traços do modelo Big Five na construção de perfis de usuários de Sistemas de Recomendação Educacionais. Particularmente, analisou-se a possibilidade de utilizar apenas algum(ns) traço(s) para simplificar esta construção, mantendo, ainda, as recomendações personalizadas acuradas. Embora não tenha sido possível destacar precisamente os traços do modelo Big Five com maior influência, os resultados apontaram que os cinco traços não têm a mesma influência na recomendação, havendo indicios de que o uso do traço Abertura seja suficiente.

\section{Introdução}

O desenvolvimento de Sistemas de Recomendação Educacionais (SRE) [Costa, Aguiar e Magalhães 2013] pode se beneficiar dos avanços da Computação Afetiva [Jaques e Nunes 2019], área que investiga, por exemplo, como os computadores podem detectar a personalidade dos indivíduos. Apesar de construtos psicológicos, tais como personalidade, estarem relacionados à tomada de decisões e, portanto, devam ser considerados ao projetar sistemas de recomendação [Tkalčič 2018], ainda é uma questão em aberto, segundo Xia et al. (2014), integrar efetivamente o fator social de personalidade em modelos de recomendação para melhorar a acurácia desses sistemas.

O modelo dos Cinco Grandes Fatores, ou Big Five, é uma versão moderna da teoria dos Traços de Personalidade (TP) [Allport e Allport 1921] — uma das várias perspectivas relacionadas à personalidade de um indivíduo. A partir desta teoria, é investigado como as pessoas são diferentes (averiguando-se quais traços melhor as descrevem) e o quanto são diferentes (averiguando-se o grau de variação nos traços) [Pervin e John 2001].

Com base no contexto exposto, considerou-se relevante a realização deste estudo, por analisar a aplicação da teoria dos TP na busca por melhorias na construção 
VIII Congresso Brasileiro de Informática na Educação (CBIE 2019)

Anais do XXX Simpósio Brasileiro de Informática na Educação (SBIE 2019)

do perfil dos estudantes (usuários) em SRE, visando a uma acurácia maior na recomendação personalizada de recursos.

Propendendo-se a simplificar a criação desse perfil dos estudantes em um SRE, o estudo descrito neste artigo objetivou investigar o impacto de utilizar apenas algum(ns) traço(s) do modelo Big Five para construir tal perfil. Portanto, as seguintes questões de pesquisa foram estabelecidas: Os TP do modelo Big Five têm a mesma influência na recomendação personalizada de recursos educacionais para estudantes? É possível destacar o(s) traço(s) com maior influência nesse processo de recomendação?

O restante deste artigo está organizado como segue: nas Seções 2 e 3, são apresentados, respectivamente, conceitos e estudos relacionados; na Seção 4, é comentado o método empregado; na Seção 5, são descritos e discutidos os resultados; na Seção 6, por fim, são realizadas algumas considerações.

\section{Fundamentação Teórica}

A Web permite facilmente que as pessoas acessem inúmeros conteúdos e lhes forneçam seus likes ou dislikes; nesse sentido, a Web tem servido como uma força motriz para o desenvolvimento de sistemas de recomendação, permitindo que as pessoas encontrem conteúdo que realmente lhes interessa [Aggarwal 2016]. Na educação, esses sistemas (SRE) podem ser usados para recomendar recursos didáticos considerando as preferências dos estudantes, auxiliando-os a alcançar determinados objetivos de aprendizagem [Costa, Aguiar e Magalhães 2013] [Ferreira, Vasconcelos e França 2017].

Uma vez que na psicologia também se estuda como um indivíduo adquire conhecimento, entende-se que os aspectos psicológicos influenciam na área da educação [Aguiar 2017]. Nesse sentido, é apropriado estudar como incorporar aspectos psicológicos em SRE, visando a melhores recomendações. Segundo Melo, Dantas e Fernandes (2017), a Computação Afetiva aplicada à educação possui o intento de prover um ambiente de aprendizagem mais afetivo ao estudante, empregando a extração de aspectos relativos à afetividade, a exemplo da personalidade.

O modelo Big Five (por vezes representado pelo acrônimo OCEAN), citado na Seção 1, define estes como sendo os cinco grandes TP: Openness to experience $(\mathbf{O})$ Abertura (ou Abertura à mudança, ou Abertura à experiência); Conscientiouness $(\mathbf{C})$ Realização (ou Conscientização, ou Conscienciosidade); Extraversion (E) Extroversão; Agreeableness (A) - Socialização (ou Amabilidade); e Neuroticism (N) - Neuroticismo (ou Instabilidade emocional) [Pervin e John 2001] [Aguiar 2017]. O uso deste modelo neste estudo justifica-se devido ao Big Five ser uma versão moderna da teoria dos TP, a teoria mais comumente aplicada para representar a personalidade [Nunes 2012 apud Aguiar 2017]. Explanações sobre outras teorias/perspectivas relativas à personalidade podem ser obtidas, por exemplo, na obra de Pervin e John (2001).

É comum a utilização de questionários/inventários para identificar informações relativas à personalidade humana, a exemplo do NEO-PI-R (Revised NEO-Personality Inventory) e do TIPI (Ten Item Personality Inventory) [Jaques e Nunes 2019]. Por outro lado, de modo a identificar os TP dos indivíduos de modo menos intrusivo como os tradicionais inventários, há pesquisas, a exemplo de [Schwartz et al. 2013], que expõem que há correlação entre TP e padrões de uso do Facebook, viabilizando a detecção dos TP dos usuários dessa rede social por meio da mineração dos dados. 
VIII Congresso Brasileiro de Informática na Educação (CBIE 2019)

Anais do XXX Simpósio Brasileiro de Informática na Educação (SBIE 2019)

Os algoritmos de Filtragem Colaborativa baseada em vizinhança — tipo de método comumente usado em sistemas de recomendação [Aggarwal 2016] — podem ser facilmente empregados em SRE, recomendando-se os recursos educacionais mais bem avaliados por usuários com preferências similares, a partir de suas avaliações prévias [Costa, Aguiar e Magalhães 2013].

Além da estratégia clássica de Filtragem Colaborativa baseada nos $K$ vizinhos/usuários que anteriormente avaliaram itens de maneira similar (UserKNN User-based K-Nearest Neighbors), é possível, por exemplo, realizar uma recomendação baseada em personalidade, usando a estratégia de identificar os vizinhos mais próximos com base nos TP [Tkalčič et al. 2009]. Alguns pesquisadores indicam em seus estudos que, em comparação aos não baseados em personalidade, os sistemas com base em personalidade possibilitam maior eficácia e lealdade dos seus usuários [Elahi et al. 2013] [Xia et al. 2014]. Além disso, os TP podem ser aplicados para resolver o problema de cold-start em sistemas de recomendação [Wu, Chen e He 2013] [Xia et al. 2014].

Ressalta-se que, este estudo, como o próprio termo Filtragem Colaborativa sugere, relaciona-se ao aspecto da colaboração, que é enfatizada nas teorias pedagógicas construtivistas, a exemplo da Teoria de Aprendizagem de Vygotsky [Vygotsky 1978].

\section{Estudos Relacionados}

Em relação a pesquisas relacionadas, em âmbito internacional, Klašnja-Milićević et al. (2017) apresentaram uma visão geral do estado-da-arte em sistemas de recomendação para sistemas de e-learning; entretanto, não mencionam TP em seu estudo. No estudo de Katarya e Verma (2016), ao categorizar sistemas de recomendação afetivos, há apenas 2 artigos classificados como 'Educacional' em relação à sua natureza. Reis et al. (2018), por sua vez, ao realizar um mapeamento sistemático sobre estado afetivo e fatores socioemocionais em Aprendizagem Colaborativa Suportada por Computador, encontraram apenas 2 artigos considerando TP relacionados a SRE. Nenhum desses 4 estudos, encontrados a partir dessas revisões internacionais, focou no objetivo considerado neste artigo.

Morais et al. (2017), em uma revisão sistemática de pesquisas publicadas no Brasil envolvendo Computação Afetiva aplicada à Educação, identificaram 7 estudos (dentre os 24 estudos selecionados na revisão) considerando o estado afetivo Personalidade; apenas 2 utilizaram o modelo Big Five, e nenhum destes diretamente relacionados a SRE. Ainda em âmbito nacional, Ferreira, Vasconcelos e França (2017) selecionaram 22 estudos em um mapeamento sistemático sobre SRE no Brasil, não listando estudos envolvendo TP (modelo Big Five).

Considerando-se tais estudos de revisão da literatura, percebe-se a escassez de pesquisas relacionadas às questões definidas na Seção 1. Recentemente, foram retornados apenas cinco artigos ao utilizar a string de busca (personalit* OR personalid*) AND recom* no Portal de Publicações da Comissão Especial de Informática na Educação (http://www.br-ie.org/pub/index.php/index/search), e nenhum com enfoque no objetivo deste artigo. Percebe-se, assim, que o uso dos TP, ou parte deles, para auxiliar na recomendação de recursos para a aprendizagem, não tem sido vastamente aplicado/estudado na literatura nacional, embora seja considerado relevante (dentre os cinco artigos, está incluso aquele premiado como o melhor artigo da edição 2018 do Simpósio Brasileiro de Informática da Educação). 
VIII Congresso Brasileiro de Informática na Educação (CBIE 2019)

Anais do XXX Simpósio Brasileiro de Informática na Educação (SBIE 2019)

As pesquisas encontradas mais relacionadas com a pesquisa descrita neste artigo são [Roshchina 2012] e [Aguiar et al. 2017]. Roshchina (2012), usando Filtragem Colaborativa com um conjunto de dados no domínio de turismo, analisou que, levandose em conta as várias combinações dos traços do modelo Big Five, foram produzidas diferentes porcentagens de avaliações encontradas corretamente por usuário. Aguiar et al. (2017) não utilizaram o modelo Big Five, mas o objetivo consistiu em analisar a influência das quatro dimensões do modelo de Estilos de Aprendizagem de FelderSilverman na construção de perfis de usuários de SRE. O método empregado neste artigo (Seção 4) é similar ao descrito em [Aguiar et al. 2017].

Uma vez que não foram encontrados estudos no domínio educacional que fizeram uma investigação experimental referente à influência dos traços do Big Five, considerou-se relevante a realização/divulgação do estudo descrito nas seções seguintes.

\section{Método}

Para analisar o impacto dos TP nas recomendações, utilizou-se a Filtragem Colaborativa, empregando-se a Distância Euclidiana para o cálculo da similaridade entre os perfis dos usuários (cada perfil de usuário consistiu em um vetor numérico de tamanho até 5 , com os valores percentuais para cada TP). Foram variados os tamanhos dos vetores representativos dos usuários, retirando valores para analisar se algum traço (ou conjunto de traços) se destacava em relação a considerar todos os traços do modelo Big Five.

Assim, foram comparadas as 31 variações do algoritmo de recomendação baseada em TP, nomeados como PBR_*****, em que cada * representa uma letra do acrônimo OCEAN (apresentado na Seção 2) ou o caractere '-', para explicitar que não foi contemplado o traço em determinada variação (por exemplo: a variação nomeada PBR_ocean contempla o modelo completo e, por sua vez, a variação nomeada PBR_o-e-n indica que não há presença dos traços conscientiouness e agreeableness). $\mathrm{O}$ termo PBR foi usado como sigla para Personality-Based Recommendation.

Para analisar o desempenho (relevância dos itens recomendados) dessas 31 variações, foi utilizada a métrica NDCG (Normalized Discounted Cumulative Gain) [Aggarwal 2016]. Além dessas variações de PBR, foi analisado seu desempenho em comparação ao desempenho de uma abordagem tradicional em Filtragem Colaborativa, o UserKNN.

Uma vez que se percebeu, no conjunto de dados utilizado, que a quantidade de usuários vizinhos, da estratégia de Filtragem Colaborativa, influenciava no resultado da recomendação, decidiu-se, além de variar os TP no experimento, realizar a análise variando-se também o valor de $K$ (sendo considerados os seguintes valores: $5,10,15$, 20 e 25), tanto para as variações de PBR quanto para o UserKNN.

O conjunto de dados utilizado contempla valores para os TP (obtidos a partir da ferramenta Five Labs [Five 2015], que usa o modelo Big Five e se baseia no estudo de Schwartz et al. (2013), citado na Seção 2) e notas (ratings em uma escala de 1 a 5) de 55 usuários (graduandos e pós-graduandos em Ciência da Computação), atribuídas a 25 itens (variações de características de Objetos de Aprendizagem, segundo o padrão IEEE LOM [IEEE 2002]).

Em relação à divisão dos dados em conjuntos de treinamento e teste, foi utilizado o método Random Subsampling [Han, Kamber e Pei 2012]. Assim, o conjunto 
VIII Congresso Brasileiro de Informática na Educação (CBIE 2019)

Anais do XXX Simpósio Brasileiro de Informática na Educação (SBIE 2019)

de dados foi separado aleatoriamente, por 100 vezes, em dois subconjuntos: $33 \%$ dos dados para o conjunto de testes e $67 \%$ dos dados para o conjunto de treinamento.

Considerou-se $\alpha=5 \%$ (ou seja, 95\% de confiança) para os intervalos de confiança gerados e para os testes estatísticos realizados - ANOVA e T (paramétricos) e Kruskal-Wallis H e Mann-Whitney U (não paramétricos) [Boslaugh e Watters 2008].

\section{Resultados e Discussões}

Baseando-se no método descrito na Seção 4, foi realizada, inicialmente, uma análise a partir de uma figura contendo todos os intervalos de confiança de todas as variações ${ }^{1}$. Analisando-se separadamente os blocos relativos aos cinco valores de $K(5,10,15,20$ e 25), foi visto que as abordagens PBR foram melhores que a UserKNN e, além disso, foi percebido que o desempenho da abordagem PBR varia dependendo dos traços considerados.

Em síntese, tendo em vista o objetivo deste estudo, não foi possível identificar o(s) TP com maior influência ao observar os intervalos de confiança na figura gerada inicialmente e, mais detalhadamente, em figuras como a Figura 1.

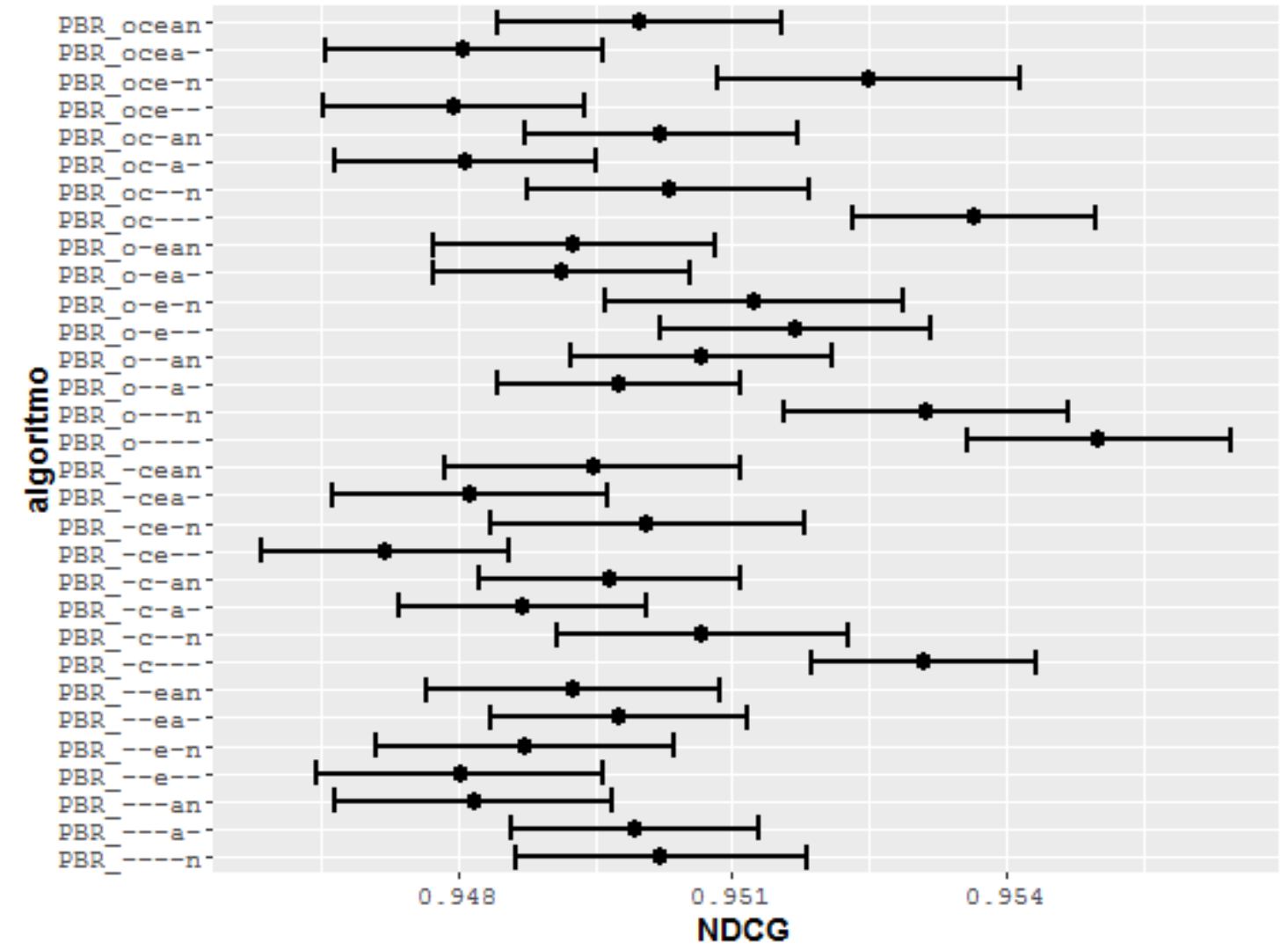

Figura 1. Intervalos de confiança para as variações do algoritmo PBR $(K=15)$.

Para confirmar o que se notou ao analisar inicialmente os intervalos de confiança, foram executados testes estatísticos, com base na blocagem relativa aos valores de $K$, e

\footnotetext{
${ }^{1}$ Decidiu-se não colocar tal figura (com 160 intervalos de confiança) neste artigo devido a seu tamanho. Todavia, a figura mencionada está disponível neste link: $<$ https://bit.ly/2YNeO8c $>$.
} 
VIII Congresso Brasileiro de Informática na Educação (CBIE 2019)

Anais do XXX Simpósio Brasileiro de Informática na Educação (SBIE 2019)

desconsiderando-se o algoritmo UserKNN. A partir dos resultados do teste paramétrico ANOVA e do teste não paramétrico Kruskal-Wallis $\mathrm{H}$, foi possível rejeitar a hipótese de que não há diferença de resultado ao variar os traços considerados nas abordagens. Isso implica dizer, em relação ao objetivo deste estudo, que os TP influenciam diferentemente na recomendação personalizada de recursos educacionais para estudantes.

Objetivando a descoberta do(s) TP com maior influência no processo de recomendação, planejou-se a definição de rankings de desempenho das variações do algoritmo PBR, para cada bloco de valores de $K$, com base nos resultados de testes $\mathrm{T}$ (paramétrico) e Mann-Whitney U (não paramétrico), par a par. Para definir esses rankings, primeiramente foi realizada uma ordenação das variações de PBR, baseandose na média da métrica NDCG; em seguida, foi realizada uma análise dos resultados de ambos os testes estatísticos ( $\mathrm{T}$ e Mann-Whitney U) para cada par de variações das posições adjacentes dessa ordenação inicial — sendo definidas posições diferentes no ranking nos casos em que ambos os testes indicassem diferença entre o par, e sendo definidas posições iguais nos casos contrários.

Utilizando-se esta abordagem, ao analisar todos os pares com os testes $\mathrm{T}$ e Mann-Whitney U, notou-se que, apesar de haver alguns pares de variação estatisticamente diferentes, não houve casos em que uma ou mais variações pudessem ser postas em uma posição diferente das demais no ranking. Considerando-se as sobreposições dos intervalos de confiança, especialmente visíveis em figuras como a Figura 1, isto era esperado. Deste modo, no Quadro 1, todas as variações empatam na mesma posição no ranking, variando a ordem com base apenas na média.

Quadro 1. Rankings de desempenho (NDCG) das variações do algoritmo PBR.

\begin{tabular}{|c|c|c|c|c|}
\hline$K=5$ & $K=10$ & $K=15$ & $K=20$ & $K=25$ \\
\hline$P B R---a-$ & $P B R---a-$ & PBR $\quad 0_{----}$ & $P B R-C---$ & $P B R---n$ \\
\hline$P B R^{-} 0---n$ & $P B R^{-} \circ--n$ & PBR ${ }^{-} \mathrm{C}^{--}-$ & $P B R^{-} 0--n$ & $P B R^{-}-C_{---}$ \\
\hline PBR $0-e^{--}$ & PBR OC--- & PBR $0---n$ & PBR $0--$ & PBR $0----$ \\
\hline$P B R_{-}---n$ & PBR_o-e-- & PBR_-C--- & $P B R=O C e-n$ & $P B R_{-}$ \\
\hline PBR_O---- & PBR o-ea- & PBR_oce-n & $P B R-C e-n$ & PBR_o-e-n \\
\hline PBR_o--a- & PBR_--e-- & PBR_o-e-- & $P B R=C--n$ & PBR_OC--n \\
\hline PBR_-c-a- & PBR_-c--n & PBR_o-e-n & $P B R \quad 0-e--$ & PBR $-c--n$ \\
\hline PBR $0 C^{-}-n$ & PBR $----n$ & $\mathrm{PBR}_{-}^{-}-\mathrm{c}--\mathrm{n}$ & PBR_o-e-n & PBR_-ce-n \\
\hline PBR_--ea- & PBR_o--a- & PBR_o--an & PBR $--e-n$ & PBR $--e-n$ \\
\hline PBR_o-e-n & PBR_OC--n & PBR_OC--n & PBR_----n & PBR_--e-- \\
\hline PBR_o-ea- & PBR_-C--- & PBR_----n & PBR_OC--- & PBR_oce-n \\
\hline PBR_oc-an & PBR_--ea- & PBR_oc-an & PBR_-c-a- & PBR_o-e-- \\
\hline PBR_oc-a- & PBR_o-ean & PBR_-ce-n & PBR_oce-- & PBR_OC--- \\
\hline PBR_o--an & PBR_OC-a- & PBR_ocean & $\mathrm{PBR}_{-}^{-}-\mathrm{C}--\mathrm{n}$ & PBR_- $-\mathrm{Ce}--$ \\
\hline PBR---an & PBR_oce-n & PBR & PBR_oc-an & PBR_oc-an \\
\hline PBR_oce-n & PBR_-c-a- & PBR & PBR_--e-- & PBR_-c-an \\
\hline PBR_-c--n & PBR_o-e-n & PBR & PBR_-ce-- & PBR_oce-- \\
\hline PBR_ocea- & PBR_--ean & PBR & $\mathrm{PBR}_{-}^{-}-\mathrm{c}-\mathrm{an}$ & PBR_O--a- \\
\hline PBR_ocean & PBR_o--an & PBR & PBR_---an & PBR_---an \\
\hline PBR_--e-n & PBR_ocean & PBR_o-ean & PBR ocea- & $\mathrm{PBR}^{-}-\mathrm{c}-\mathrm{a}-$ \\
\hline PBR_--e-- & PBR_-ce-n & PBR & PBR_o--an & PBR_---a- \\
\hline PBR_-ce-n & PBR_oc-an & PBR_o-ea- & PBR_--ea- & PBR_o--an \\
\hline $\mathrm{PBR}_{-}^{-}-\mathrm{c}-\mathrm{an}$ & PBR_oce-- & PBR_--e-n & PBR_o--a- & PBR_ocea- \\
\hline PBR_o-ean & PBR_--e-n & PBR_-c-a- & PBR_---a- & PBR_-cean \\
\hline PBR_OC--- & PBR_-cea- & PBR_---an & PBR_o-ea- & PBR_--ean \\
\hline PBR_oce-- & PBR_O---- & PBR_-cea- & PBR_-cea- & PBR_oc-a- \\
\hline PBR_-cean & PBR_-c-an & PBR_OC-a- & PBR_--ean & PBR_o-ean \\
\hline PBR_--ean & PBR_-cean & PBR_ocea- & PBR_-cean & PBR_--ea- \\
\hline PBR_-C--- & PBR_ocea- & PBR_--e-- & PBR_o-ean & PBR_o-ea- \\
\hline PBR_-cea- & PBR_-ce-- & PBR_oce-- & PBR_ocean & PBR_-cea- \\
\hline PBR - $\mathrm{Ce}^{--}$ & PBR_---an & PBR_-ce-- & PBR_oc-a- & PBR_ocean \\
\hline
\end{tabular}


VIII Congresso Brasileiro de Informática na Educação (CBIE 2019)

Anais do XXX Simpósio Brasileiro de Informática na Educação (SBIE 2019)

No Quadro 1, está realçada a variação PBR_ocean (que considera todo o modelo Big Five), sendo destacadas em negrito as variações com resultados similares (isto é: nos testes par a par, o p-valor obtido para as variações que estão em negrito e a variação PBR_ocean foi maior que o nível de significância de 5\%, não sendo possível rejeitar a hipótese nula de que, em média, as variações são iguais em relação à métrica NDCG; logo, não se considerou diferença entre os pares). Em itálico, e agrupadas por chaves, estão as variações com maior acurácia na recomendação em comparação com a variação que considera todos os cinco traços do modelo (isto é: pelos testes par a par, considerou-se diferença entre a variação PBR_ocean e as variações em itálico).

Para $K=5$, as variações $\mathrm{PBR}_{----\mathrm{a}-}$, PBR_o---n, PBR_o-e-- e PBR_----n apresentaram-se, com base nos testes par a par, melhores que $\mathrm{PBR} \_$ocean, que teve o $19^{\circ}$ maior valor médio. Para $K=10$, a variação $\mathrm{PBR} \_$ocean teve o $20^{\circ}$ maior valor médio, apresentando, com base nos testes par a par, desempenho inferior às variações PBR_---a-, PBR_o---n, PBR_oc---, PBR_o-e-- e PBR_o-ea-. Para $K=15$, a variação PBR_ocean teve o $14^{\circ}$ maior valor médio, sendo percebido, a partir dos testes par a par, que a variação PBR_o---- apresentou-se melhor que ela. Para $K=20$, a variação PBR_ocean teve o segundo menor valor médio, apresentando, a partir dos testes par a par, resultado inferior em relação às variações $P B R_{-} \mathrm{C}^{---}, \mathrm{PBR} \mathrm{O}^{---n}$, $\mathrm{PBR} \mathrm{O}^{-----}$, PBR_oce-n, PBR_-ce-n, PBR_oc--n, PBR_o-e-- e PBR_o-e-n. Para $K=25$, a variação PBR_ocean teve o menor valor médio, apresentando, a partir dos testes par a par, resultado inferior em relação às variações $P B R_{----n}, P_{-} R_{-C^{--}}, P^{-} R_{-}{ }^{----} \mathrm{e}$ PBR_o---n.

Com isso, percebe-se a dificuldade em indicar precisamente o(s) traço(s) com maior influência na acurácia das recomendações baseadas em TP. Ressalta-se que, apesar do ranking para $K=5$ apresentar algumas variações (PBR_-c---, PBR_-cea- e PBR_-ce--) com desempenho mais baixo que a variação com todos os traços (PBR_ocean), as outras variações não tendem a diminuir significativamente o desempenho do PBR_ocean.

Embora nenhuma variação tenha se destacado em todos os rankings, há variações que tendem a ter melhores desempenhos que PBR_ocean: a variação PBR_o---n (com desempenho melhor que PBR_ocean nos rankings de $K$ igual a 5, 10,

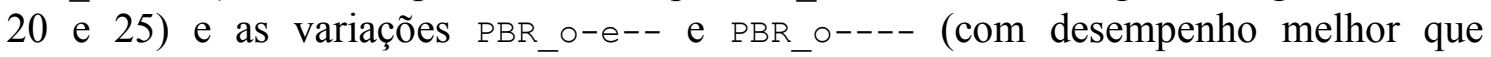
PBR_ocean em pelo menos três dos rankings). Isso indicia a importância do traço Openness to experience (Abertura) do modelo Big Five. Vale ressaltar que, no geral, o desempenho das recomendações foi melhor com $K=25$ e $K=20$ em comparação a $K=5$ e $K=10$, por exemplo. Portanto, nota-se que o traço Abertura individualmente (PBR_o----) tende a ter desempenho melhor que a combinação de todos os traços (PBR_ocean).

No estudo de Roshchina (2012), a autora concluiu, com seus experimentos, que a combinação de Abertura, Realização, Extroversão e Neuroticismo (equivalente ao que foi denominado neste artigo como $\mathrm{PBR}$ oce-n) possibilitou a maior porcentagem de avaliações encontradas corretamente por usuário, seguida pela combinação de todas as cinco características. Embora o estudo de Roshchina (2012) use um conjunto de dados de um domínio não educacional, a combinação $P B R \_o c e-n$ ter desempenho melhor que a combinação PBR_ocean é vista também no ranking relativo ao $K=20$ do Quadro 1 . 
VIII Congresso Brasileiro de Informática na Educação (CBIE 2019)

Anais do XXX Simpósio Brasileiro de Informática na Educação (SBIE 2019)

\section{Considerações Finais}

Visando responder às questões de pesquisa expostas na Seção 1, os resultados obtidos indicaram, primeiramente, que os traços do modelo Big Five influenciam de maneira diferente na recomendação personalizada de recursos educacionais para estudantes; em seguida, percebeu-se não haver um destaque expressivo nos traços, para, por exemplo, desconsiderar os demais traços na utilização do modelo Big Five em SRE. Contudo, a partir da avaliação experimental realizada, observou-se que considerar apenas o traço Abertura seria suficiente (tão acurado quanto considerar todos os cinco traços), podendo tal traço inclusive influenciar na obtenção de maior acurácia das recomendações.

Considerar a não totalidade do modelo Big Five ao construir o perfil do usuário em SRE implicaria, por exemplo, em menos questões necessárias dos inventários de personalidade para os usuários responderem, ou, por outro lado, poderia implicar na simplificação do projeto de regras em sistemas com detecção automática. Vale ressaltar que, com a base de dados utilizada, as variações do algoritmo de recomendação baseada em TP apresentaram desempenho melhor que o UserKNN. Como estudo futuro, é conveniente aprofundar este estudo da influência dos TP utilizando outros conjuntos de dados educacionais, a fim de comparar resultados e reanalisar a possibilidade de simplificar o uso do algoritmo PBR em SRE, focando, se for o caso, no traço Abertura do modelo Big Five.

Com o objetivo de examinar a influência das dimensões do Big Five no comportamento de compartilhamento de conhecimento dos indivíduos (acadêmicos de uma universidade pública), Lotfi et al. (2016) concluíram em um estudo que os TP são uma característica importante que influencia o compartilhamento de conhecimento. Os autores indicaram que os traços Abertura, Extroversão e Realização têm influência significativamente positiva no comportamento de compartilhamento de conhecimento dos indivíduos. Apesar de tal estudo não ter foco em Sistemas de Recomendação, é curioso perceber que os autores indicaram em seus resultados que o traço Abertura parece ser o fator mais significativo que influencia a partilha de conhecimento. Além disso, uma vez que os resultados de Lotfi et al. (2016) indicaram que os TP são uma característica com influência no compartilhamento de conhecimento, isto corrobora com a importância de se estudar como realizar, por meio da Filtragem Colaborativa, recomendação de recursos educacionais usando a teoria dos traços.

A relevância do estudo descrito neste artigo não consiste em indicar que se deve ou não usar a teoria dos TP desconsiderando algum traço, mas ressalta-se a possibilidade de considerar que determinados traços podem ter maior influência no processo de recomendação em SRE, podendo-se pensar em ponderá-los.

A Computação Afetiva, ao investigar cientificamente aspectos psicológicos no âmbito educacional, é um campo promissor para possibilitar ainda mais o uso das Tecnologias Digitais de Informação e Comunicação visando à melhoria da educação [Aguiar 2017]. No Brasil, ainda são poucos os artigos/autores que estudam essa área [Jaques e Nunes 2019]. Com a divulgação deste estudo, que aborda a teoria de TP em SRE, espera-se contribuir com a linha de pesquisa relativa aos estudos de soluções que envolvem personalização de ambientes computacionais para a promoção da aprendizagem. 
VIII Congresso Brasileiro de Informática na Educação (CBIE 2019)

Anais do XXX Simpósio Brasileiro de Informática na Educação (SBIE 2019)

\section{Agradecimento}

À CAPES (Coordenação de Aperfeiçoamento de Pessoal de Nível Superior), pelo apoio financeiro.

\section{Referências}

Aggarwal, C. C. (2016). Recommender Systems: The Textbook. 1 ed. Springer International Publishing, 498 p., ISBN: 978-3-319-29659-3. doi:10.1007/978-3-319-29659-3

Aguiar, J. J. B. (2017). Considerando Estilos de Aprendizagem, Emoções e Personalidade em Informática na Educação. Informática na Educação: teoria \& prática, Porto Alegre, v. 20 , n. 2, p. $85-102$. doi:10.22456/1982-1654.65333

Aguiar, J. J. B.; Barbosa, A. F.; Araújo, J. M. F. R.; Costa, E. B. (2017). Um Estudo sobre a Influência das Dimensões do Modelo Felder-Silverman na Recomendação de Recursos Educacionais baseada nos Estilos de Aprendizagem dos Alunos. In: Anais do Simpósio Brasileiro de Informática na Educação (SBIE), Recife, p. 1277-1286. doi:10.5753/cbie.sbie.2017.1277

Allport, F. H.; Allport, G. W. (1921) Personality Traits: Their Classification and Measurement. The Journal of Abnormal and Social Psychology, v. 16, n. 1, p. 6-40. doi: $10.1037 / \mathrm{h} 0069790$

Boslaugh, S.; Watters, P. A. (2008). Statistics in a Nutshell. 1st ed. O'Reilly Media, Inc., Sebastopol, CA, USA. ISBN: 978-0-596-51049-7.

Costa, E.; Aguiar, J.; Magalhães, J. (2013). Sistemas de Recomendação de Recursos Educacionais: conceitos, técnicas e aplicações. In: Anais da Jornada de Atualização em Informática na Educação (JAIE), p. 57-78. http://br-ie.org/pub/index.php/pie/article/view/2589

Elahi, M.; Braunhofer, M.; Ricci, F.; Tkalcic, M. (2013). Personality-Based Active Learning for Collaborative Filtering Recommender Systems. In: Baldoni, M.; Baroglio, C.; Boella, G.; Micalizio, R. (Eds.), Proceeding of the XIIIth International Conference on AI*IA 2013: Advances in Artificial Intelligence, v. 8249, p. 360-371, Springer. doi:10.1007/978-3-319-03524-6_31

Ferreira, V. A. S.; Vasconcelos, G. C.; França, R. S. (2017). Mapeamento Sistemático sobre Sistemas de Recomendações Educacionais. In: Anais do Simpósio Brasileiro de Informática na Educação (SBIE), Recife, p. 253-262. doi:10.5753/cbie.sbie.2017.253

Five. (2015). Five Labs - See the personality behind your posts. Product of Five.com. Disponível em: $<\mathrm{http}: / /$ labs.five.com/>. Acesso em: $24 \mathrm{abr}$. 2015. [The tool is no longer supported]

Han, J.; Kamber, M.; Pei, J. (2012). 8 - Classification: Basic Concepts. In: Data Mining: Concepts and Techniques (Third Edition) - A volume in The Morgan Kaufmann Series in Data Management Systems, p. 327-391, ISBN: 978-0-12-381479-1. doi:10.1016/B978-0-12-381479-1.00008-3

IEEE. (2002). IEEE Standard for Learning Object Metadata. New York, USA: IEEE Computer Society (The Institute of Electrical and Electronics Engineers, Inc.). 32 p. IEEE Std 1484.12.1-2002. doi:10.1109/IEEESTD.2002.94128

Jaques, P. A.; Nunes, M. A. S. N. (2019). Computação afetiva aplicada à educação. In: Pimentel, M.; Sampaio, F. F.; Santos, E. O. (Org.). Informática na Educação: técnicas e tecnologias computacionais. Porto Alegre: SBC. (Série Informática na Educação, v. 3.). Disponível em: <http://ieducacao.ceie-br.org/computacaoafetiva $>$. Acesso em: 07 jul. 2019.

Katarya, R.; Verma, O. P. (2016). Recent developments in affective recommender systems. 
VIII Congresso Brasileiro de Informática na Educação (CBIE 2019)

Anais do XXX Simpósio Brasileiro de Informática na Educação (SBIE 2019)

Physica A: Statistical Mechanics and its Applications, v. 461, p. 182-190. doi:10.1016/j.physa.2016.05.046

Klašnja-Milićević, A.; Vesin, B.; Ivanović, M.; Budimac, Z.; Jain, L. C. (2017). E-Learning Systems: Intelligent Techniques for Personalization. Springer International Publishing. ISBN: 978-3-319-41163-7. doi:10.1007/978-3-319-41163-7

Lotfi, M.; Muktar, S. N. B; Ologbo, A. C.; Chiemeke, K. C. (2016). The Influence of the Big-Five Personality Traits Dimensions on Knowledge Sharing Behavior. Mediterranean Journal of Social Sciences, v. 7, n. 1 S1, p. 241-250. doi:10.5901/mjss.2016.v7n1s1p241

Melo, S.; Dantas, A. C.; Fernandes, M. (2017). Modelo do estudante baseado em emoções e perfis de personalidade para recomendação de estratégias pedagógicas personalizadas. In: Anais do Simpósio Brasileiro de Informática na Educação (SBIE), Recife, p. 967976. doi:10.5753/cbie.sbie.2017.967

Morais, F.; Silva, J.; Reis, H.; Isotani, S.; Jaques, P. (2017). Computação Afetiva aplicada à Educação: uma revisão sistemática das pesquisas publicadas no Brasil. In: Anais do Simpósio Brasileiro de Informática na Educação (SBIE), Recife, p. 163-172. doi:10.5753/cbie.sbie.2017.163

Pervin, L. A.; John, O. P. (2001). Handbook of Personality: Theory and research. Guilford Press, New York. ISBN: 9781572306950.

Reis, R. C. D.; Isotani, S.; Rodriguez, C. L.; Lyra, K. T.; Jaques, P. A.; Bittencourt, I. I. (2018). Affective states in computer-supported collaborative learning: Studying the past to drive the future. Computers \& Education, v. 120, p. 29-50. doi:10.1016/j.compedu.2018.01.015

Roshchina, A. (2012). TWIN: Personality-based Recommender System. MSc thesis. Tallaght Institute of Technology, Ireland. Disponível em: $<$ http://users.dsic.upv.es/ prosso/resources/RoshchinaMSc.pdf $>$. Acesso em: 07 jul. 2019.

Schwartz, H. A.; Eichstaedt, J. C.; Kern, M. L.; Dziurzynski, L.; Ramones, S. M.; Agrawal, M.; Shah, A.; Kosinski, M.; Stillwell, D.; Seligman, M. E. P.; Ungar, L. H. (2013). Personality, Gender, and Age in the Language of Social Media: The Open-Vocabulary Approach. PLoS ONE, v. 8, n. 9, p. e73791. doi:10.1371/journal.pone.0073791

Tkalčič, M. (2018). Emotions and Personality in Recommender Systems: Tutorial. In: Proceedings of the 12th ACM Conference on Recommender Systems (RecSys'18), Vancouver, p. 535-536. doi:10.1145/3240323.3241619

Tkalčič, M.; Kunaver, M.; Tasič, J.; Košir, A. (2009). Personality Based User Similarity Measure for a Collaborative Recommender System. In: Proceedings of the 5th Workshop on Emotion in Human-Computer Interaction-Real World Challenges, p. 30-37.

Vygotsky, L. (1978). Mind in Society: The Development of Higher Psychological Processes. Harvard University Press, Cambridge, MA.

$\mathrm{Wu}, \mathrm{W}$.; Chen, L.; He, L. (2013). Using personality to adjust diversity in recommender systems. In: Proceedings of the 24th ACM Conference on Hypertext and Social Media (HT '13). ACM, New York, NY, USA, p. 225-229. doi:10.1145/2481492.2481521

Xia, F.; Asabere, N. Y.; Liu, H.; Chen, Z.; Wang, W. (2014). Socially Aware Conference Participant Recommendation with Personality Traits. IEEE Systems Journal, p. 1-12. doi:10.1109/JSYST.2014.2342375 